Discussion: Frequently, the public health emergency operation center in an affected country is not able to obtain the critical information of an affected area in the acute phase of disasters. This HNA tool would be used in the acute phase by the Emergency Medical Teams (EMTs) because the EMT has mobility and workforce for assisting the affected country. We have agreed on the usage of the assessment form as a kind of an "interview guide". The purpose of this assessment form is to assess a disaster situation. The next step will be to provide more opportunities for the ASEAN member states to use and learn more about this HNA form.

Prehosp Disaster Med 2019;34(Suppl. 1):s134-s135

doi:10.1017/S1049023X19002942

HESPER SW: A Web-Based Tool to Assess Needs

Dr. Karin Hugelius ${ }^{1}$, Dr. Maya Semrau ${ }^{2}$, Dr. Marie Holmefur ${ }^{1}$

1. Örebro Univeristy, Orebro, Sweden

2. King's Collage, London, Great Britain

Introduction: The Humanitarian Emergency Settings Perceived Needs Scale (HESPER) evaluates experienced needs among disaster-affected populations and has been frequently used in both humanitarian emergencies and research. Today, the use of this tool is increasing among people affected by crises and emergencies. Web-based methods have shown to reduce several methodological and practical challenges in disaster health research.

Aim: This project aims to develop and evaluate HESPER SW (a self-administered, web-based version of the HESPER scale). Methods: Alternative reliability and test-retest validity of HESPER SW were evaluated using different analytic statistical methods.

Results: The first analysis suggests that HEPSER SW is a reliable and valid instrument which is easy to use and that it reduces several methodological and practical challenges in disaster health research.

Discussion: HESPER SW can be used both for humanitarian and research purposes and offers a quick, self-administered, web-based, and scientifically robust way to investigate experienced needs in populations affected by disasters or humanitarian crises.

Prehosp Disaster Med 2019;34(Suppl. 1):s135

doi:10.1017/S1049023X19002954

\section{The HOPE Model for Disaster Nursing} Dr. Karin Hugelius, Dr. Annsofie Adolfsson

Örebro Univeristy, Orebro, Sweden

Introduction: Despite a large number of nurses involved in disaster situations in different ways, there are few theories or models that define and describe the goal and content of disaster nursing.

Aim: This study aimed to present a model for disaster nursing, based on a literature review of the concept and content of disaster nursing.
Methods: A systematic literature review of 15 original qualitative or quantitative articles was conducted. A thematic synthesis was used to analyze the data.

Results: The main theme of Disaster Nursing: Crossing Borders, included three dimensions (personal borders, professional borders and environmental borders) and four themes describing the process of disaster nursing (being hit by reality; adapting to the conditions; providing aid, relief, and caring; recovering, remembering, and growing). Based on these results the HOPE model was developed. 'HOPE' stands for 'Holistic health assessment and promotion; Organization and management of immediate response; Professional adaptation; Endurance and recovery.

Discussion: The HOPE model for disaster nursing describes the core element and essence of nursing in the disaster response phase and can serve as guidance both for nurses deployed in disasters and in disaster nursing training.

Prehosp Disaster Med 2019;34(Suppl. 1):s135

doi:10.1017/S1049023X19002966

\section{Hospital C.O.D.E (Clinical, Operational, Disaster, and Emergency) Terminology Christine Buckley ${ }^{1,2}$, Dr. Michael S. Molloy ${ }^{1,3}$, Dr. Alexander Hart ${ }^{1,2}$, Amalia Voskanyan ${ }^{1,2}$, Dr. Ritu Sarin ${ }^{1,2}$, A Prof. Gregory Ciottone $e^{1,2}$ \\ 1. BIDMC Fellowship in Disaster Medicine, Boston, United States \\ 2. Department of Emergency Medicine Beth Israel Deaconess Medical Center, Boston, United States \\ 3. University College Dublin, Belfield, Dublin, Ireland}

Introduction: Healthcare facilities frequently use disaster codes as a way to communicate with employees that an emergency or incident is occurring. As increasing numbers of providers work at multiple facilities, and healthcare systems continue to build disaster response teams and protocols covering multiple facilities, standardization of disaster code terminology is critical. A lack of consistency in terminology can potentially have a devastating impact on the understanding and response of visiting or relief staff.

Aim: To evaluate the level of standardization in terminology of disaster codes in healthcare facilities.

Methods: A convenience sample was taken from a private Facebook $^{\mathrm{TM}}$ group consisting of emergency department nurses from a wide range of facilities. The Facebook ${ }^{\mathrm{TM}}$ group was asked to share their hospital disaster codes. Of the 40,179 total members, 78 commented, including 55 photos of quick reference badges, and the rest were descriptions/lists of codes. One badge was excluded due to a blurry photograph. Results were collated and analyzed for trends and standardization.

Results: The most common codes were, "Code Red" for fire (72.7\%), "Code Blue" for cardiac arrest (44.9\%), "Code Silver" for active shooter/weapons event (37.7\%) and "Code Orange" for hazardous materials (33.8\%). There were 168 instances of a code term being associated with a particular event by five or fewer facilities. Two facilities used numeric systems, with 11 using plain language descriptions. 
Discussion: Disaster code language is inconsistent. Few of the codes were consistently assigned to the same meaning, and none were universal. Color coding was the most common method, but there was little consistency even within color code systems. Additionally, some facilities used a combination of colors, numbers, terms, and plain language. Healthcare facilities should embrace standard terminology and create a consistent language for disaster codes to enhance response capabilities and medical security.

Prehosp Disaster Med 2019;34(Suppl. 1):s135-s136

doi:10.1017/S1049023X19002978

How Antibiotic Resistance Impacts Responses to Public Health Emergencies and Strategies to Mitigate the Impacts Ms. Katbrine Robnett

Pennsylvania State University, University Park, United States

Introduction: Antibiotic resistance is when bacteria change and adapt in response to antibiotics, becoming able to defeat these drugs when used to treat infections. A direct consequence of this adaptation is an increased difficulty in treating multiple diseases. Because of increased antibiotic resistance, the World Health Organization has declared it a significant threat to public health. Aim: One frequent consequence of natural disasters is infections, as seen in the December 2004 Indian Ocean tsunami. Survivors sustained a variety of wound infections that ranged from common pathogens to rarely seen organisms including fungi.

Methods: This research analyzes the microbiology observed in wound infections associated with exposure to freshwater, seawater, soil, fecal, and other contamination after Hurricane Harvey in 2017 and Hurricane Florence in 2018.

Discussion: Therapies for infections will also be discussed in addition to how the utilization of rapid detection technology for antimicrobial resistance and correct treatments require antimicrobial susceptibility knowledge to improve health outcomes, lower economic costs, prevent further spread of multi-drug resistant outbreaks and assist with antimicrobial stewardship.

Prehosp Disaster Med 2019;34(Suppl. 1):s136

doi:10.1017/S1049023X1900298X

How to Shorten the Rescue Time in Marathon by Using BLE Communication Devices: A New Study for the EMS System in Taiwan

Dr. Kuo-song Chang $1,2,3,4, M r$. Sun Chun-I ${ }^{4}$, Professor Jung-

Tang Huang ${ }^{4}, M r$. Shib-Chi Weng ${ }^{5}, M r$. Meng-Fan Chien

1. Mackay Memorial Hospital, Taipei, Taiwan

2. MacKay Medical College, Taipei, Taiwan

3. Mackay Junior College of Medicine, Nursing, and Management, Taipei, Taiwan

4. College of Mechanical \& Electrical Engineering, National Taipei University of Technology, Taipei, Taiwan

5. Institute of Mechatronic Engineering, National Taipei University of Technology, Taipei, Taiwan

Introduction: More than one million runners have joined the marathon games since 2007 in Taiwan. There were over 150 marathon games held in Taiwan in 2018. The increase rate was $21 \%$ as compared to that of 2014 . The medical encounter rate was $1.33 \%$ in 2015 and increased to $1.41 \%$ in 2017 . The most common type of injury was muscle spasm. The second most common was abrasion due to falls. The treatment for muscle spasm was RICE only. Cardiac arrest of marathon runners was reported occasionally and time is critical for rescue.

Aim: To shorten the rescue time of the runners in an emergency. Base on the prodromal research, BLE communication technology is further used to improve the rescue positioning communication technology in the marathon.

Methods: After rescue notification devices have been set up in each $0.5 \mathrm{~km}$ on the runway of the marathon, the runner can send a rescue signal through the rescue notification devices in case of emergency. The rescue signal, periodically advertisement SN\# with rescue mark, of the runner can be precisely located and the rescue can be started very soon.

Results: In the simulation, the rescue signal can be located in 7.5 minutes, fastest in 3 seconds. The precision rate of timing is $\pm 160 \mathrm{~ms} / 6 \sigma$ that under IAAF accuracy requirement. The location error is less than 20 meters, and the rescue time can be shortened to one half as before.

Discussion: The rescue time of runner is correlated with the quality of marathon EMS. It is critical to the runner, especially in cardiac arrest. By using BLE communication devices, the runner can be located faster and more precisely. As rescue time shortened, CPR \& AED can be given sooner. The quality of marathon EMS will be improved substantially.

Prehosp Disaster Med 2019;34(Suppl. 1):s136

doi:10.1017/S1049023X19002991

The Human Disaster and the Urgency of the Intersetorial Join Between Public Social Policies: Lives that are Lost and Stories that Repeat Themselves

A/Prof. Maria Isabel Barros Bellini, Me. Nadianna Marques

Pontificia Universidade Católica/PUCRS, Escola De Saude Pública/ ESP/SES, Porto Alegre, Brazil

Introduction: The human disaster is a permanent challenge for the Brazilian government because the difficulties faced are related to the lack of interface between public policies, resulting in fragile analyses of risk and non-prevention, being that annually several Brazilian lives are lost in disasters that continue to happen.

Aim: The article is the result of research and presents the analyses of health policy, actions, and programs developed to anticipate the fire victims of the Nightclub Kiss concert hall that took place in 2013 (Santa Maria, Brazil). The objective was to investigate and analyze the disasters and human disasters, especially the fire of Nightclub Kiss when 242 young people died. Causes and determinants were analyzed in order to subsidize public policies, in particular, the health policy.

Methods: A qualitative case study supported by the critical dialectic method with semi-structured interviews, focus group, documentary analysis, and bibliographic review.

Results: The experiences accumulated throughout history show that disaster situations require public policies to be able to act readily, resolve, and pay attention to the needs of the population involved. Disasters are increasingly recurrent episodes and 